\title{
Volvulus of the Cecum Due to Intestinal Malrotation in an Adult Woman, a Case Report
}

\section{Buendía-García Ana Laura ${ }^{1}$, Bizueto-Rosas Héctor ${ }^{2 *}$, Hernández-Pérez Noemí Antonia ${ }^{3}$, Bizueto-Blancas Noelly Noemí, Pérez-González Hugo Alonso ${ }^{5}$, Gómez-Calvo Carlos Daniel ${ }^{6}$, Jiménez-Canet-Atilano Alejandro ${ }^{1}$, Caltenco-Solís Raúl Beder ${ }^{1}$, Mijangos-Montaño Ared ${ }^{1}$ and Radilla-Flores Mariana ${ }^{1}$}

${ }^{1}$ Senior Resident, Department of General Surgery, Instituto de Seguridad y Servicios Sociales de los Trabajadores del Estado, México ${ }^{2}$ Specialist, Department of Vascular and General Surgery, Instituto de Seguridad y Servicios Sociales de los Trabajadores del Estado, México

${ }^{3}$ Specialist, Department of Work and Family, Hospital General Dr. Darío Fernández Fierro, Instituto de Seguridad y Servicios Sociales de los Trabajadores del Estado, México

${ }^{4}$ Medical Student, St. Luke Medical School, Instituto de Seguridad y Servicios Sociales de los Trabajadores del Estado, México

${ }^{5}$ Specialist, Department of Vascular Surgery, Hospital General Regional, Querétaro, Instituto de Seguridad y Servicios Sociales de los Trabajadores del Estado, México

${ }^{6}$ Specialist, Department of Vascular Surgery, Hospital General Regional, Ensenada, Instituto Mexicano del Seguro Social, México

* Corresponding author: Bizueto-Rosas Héctor, Specialist, Department of Vascular and General Surgery, Instituto de Seguridad y Servicios Sociales de los Trabajadores del Estado, México, E-mail: dr_bizueto_h@yahoo.com

Received: 28 May, 2019 | Accepted: 07 Jun, 2019 | Published: 10 Jun, 2019

Citation: Buendía-García AL, Bizueto-Rosas H, Hernández-Pérez NA, Bizueto-Blancas NN, Pérez-González HA, et al. (2019) Volvulus of the Cecum Due to Intestinal Malrotation in an Adult Woman, a Case Report. J Surg Open Access 5(2): dx.doi.org/10.16966/2470-0991.181

Copyright: (C) 2019 Buendía-García AL, et al. This is an open-access article distributed under the terms of the Creative Commons Attribution License, which permits unrestricted use, distribution, and reproduction in any medium, provided the original author and source are credited.

\begin{abstract}
We present the case of an adult woman with a medical history of repetitive episodes of intestinal subocclusion and occlusion that remitted with medical treatment. During hospital admission due to acute abdominal pain and secondary to CT scan results, an urgent surgical approach was decided with findings of a malrotation anomaly consisting in a short, dilated and volvulus cecum behind hepatoduodenal ligament and a collapsed transversal colon, requiring intestinal resection and ileocolic primary intestinal anastomosis.

Conclusion: Repetitive intestinal occlusion and subocclusion symptoms also in an adult patient with history of repetitive episodes that were always treated conservatively should be considered as a possible malrotation anomaly. Cecal volvulus is a very rare clinical entity, difficult to early diagnose and can be associated to a malrotation anomaly. Early surgery in this types of patients, that are used to conservative treatment, require a high index of diagnostic suspicion but can be associated with lower mortality and morbidity.
\end{abstract}

Keywords: Intestinal occlusion; Cecal volvulus; Intestinal malrotation; Adult patient

\section{Introduction}

Intestinal malrotation is due to the interruption of the normal rotation process of the gastrointestinal tract. Embryological gut developments initially occur between the fourth and fifth week of gestation; the primitive intestine divides into two loops, proximal and distal, according its relation to the superior mesenteric artery. These two loops rotate about 270 grades, independently, anti-clockwise and then adhere to the peritoneum. Interruption of this rotation process is known as a malrotation anomaly. This congenital rotation and intestine fixation anomaly it is usually presented during the neonatal period. Infrequently they can be diagnosed in older pediatric patients or adults, requiring imaging studies to confirm the diagnosis [1]. Malrotation anomalies complications usually are intestinal occlusion due to volvulus, defined as an abnormal degree of intestinal rotation along the axis of its mesentery.
Less frequently exists, other causes such as the Ladd bandages, defined as "congenital reinforcements of the posterior peritoneum, constitute a fibrotic band from the lower liver portion up to the mesentery origin, so it surrounds and usually obstructs duodenum or other intestinal portions." Intestinal malrotation can be associated to other entities, such as ureter or kidney agenesis, extrahepatic biliary atresia, congenital diaphragmatic hernia, intestinal or duodenal stenosis or atresia, Hirschprung disease, pyloric stenosis, Prune-Belly disease or heterotaxia [1-3], or associated to duodenal invagination or to appendicitis [4].

In the adult population, it is an undiagnosed entity with an unknown incidence. Kantor studied 2000 asymptomatic patients with a barium enema and stated an incidence of intestinal malrotation of $0.19 \%$ [5]. Imaging studies, such as Doppler ultrasound can establish diagnosis by evaluation of the mesenteric vessels, normal 
vein position in front of inferior cava, and normal arterial position anterior and left of the aorta. Also with more specific signs, such as the Whirlpool sign, finding of the superior mesenteric vein, intestine and its mesentery rotate surrounding the artery and locates the intestinal volvulus. Although it has low sensitivity and specificity due to be observer dependent [1]. Abdominal CT scan can show a highabdominal obstruction, gastric and duodenum dilation and a "CT whirlpool sign" described by Fischer in 1981. CT scan can also show signs of having intestinal structures to the right, and colonic structures to the left, no uncinated pancreatic process [6], and a high-cecum [3], this being normal in up to $20 \%$ of patients.

Intestinal transit test can demonstrate as intestinal malrotation findings a small intestine to the right and the jejunum-duodenum union not crossing to the left of the spinal column; also jejunum volvulus and a corkscrew duodenum [3].

Treatment by Ladd surgical procedure consists in volvulus unfolding, non-viable intestinal resection, peritoneum bandages section, usually appendectomy and intestinal structures accommodation, having small intestine to the right and colonic structures to the left $[1,3]$. When early diagnosed, as in most cases is, the laparoscopic approach is an effective and minimally invasive procedure that allows abdominal direct vision $[2,3]$. Ladd bandages section and duodenum liberation using bipolar diathermy is usually simple [7].

\section{Case Report}

An adult woman of 65 years of age, with a medical history of constipation with bowel movements every 3 to 4 days, managed with a high-fiber diet and high-liquid intake. Medical record of several Emergency Department admissions due to intestinal occlusion and subocclusion symptoms requiring conservative management and hospital discharge in the last years.

Two days prior to last admission presents severe abdominal pain, oppressive, described as a 8/10 of intensity, nausea, vomit, without evacuations or intestinal gas channeling so a CT scan was required; it reported stomach and small intestinal as normal, colon with a highileocecal union next to the liver, abnormal mesenteric rotation, and altered morphology of ascendant colon and cecum, with a localized colon distension up to $6.5 \mathrm{cms}$. Sigmoid colon with diverticulum but no inflammatory signs; with the CT scan conclusion of images suggestive for

1. Colon anomaly with no clear presence of abdominal colon due to its high-location behind liver and volvulus, and

\section{Volvulus of the cecum (Figure 1).}

Received in our medical institution with dehydration, a nasogastric tube obtaining gastric residual, tachycardia up to 110 beats per minute, abdominal distension, pain and tympanic percussion. Diminished peristaltic noise, metallic noise in upper abdominal quadrants, and acute abdominal signs present (Von Blumberg and McBurney). Initial blood test with leukocytosis of 12,000 and 75\% of neutrophil count.

Considering clinical and CT scan findings, medical record of past repetitive abdominal pain and intestinal occlusion symptoms, an abdominal laparotomy was required. Reported findings of important gastric distension, $20 \mathrm{cms}$ long normal appendix, Ladd peritoneal bandages from the liver to the cecum prior its path behind the hepatoduodenal ligament, and posterior of which, an intestinal dilated segment up to $12 \mathrm{cms}$ of diameter, with firm fibrotic bandages to stomach and duodenum, with transverse colon and ascendant colon collapsed behind the posterior part of the hepatoduodenal ligament (Figures 2 and 3).

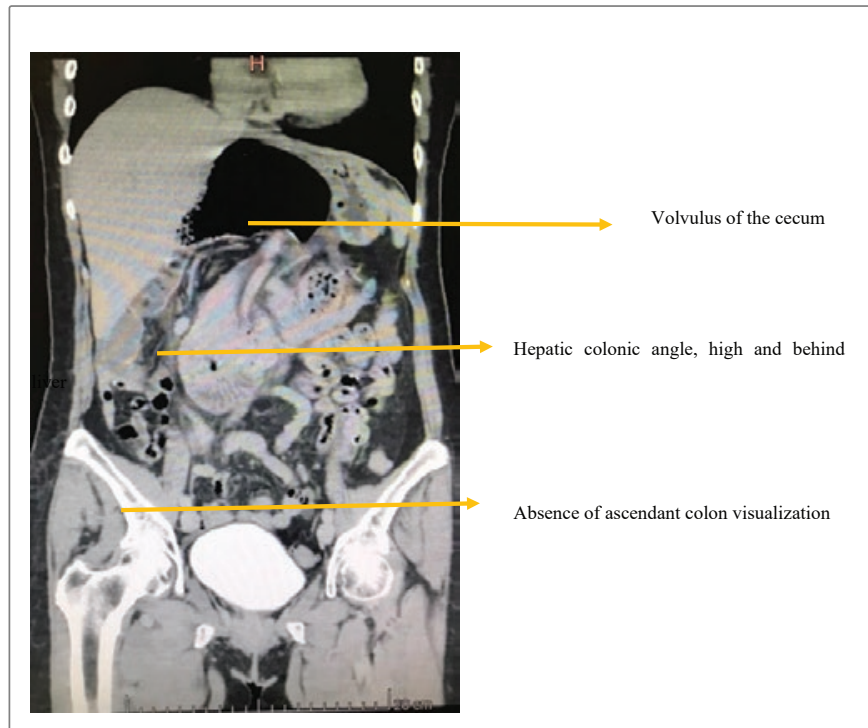

Figure 1: Abdominal CT scan, coronal image. No ascendant colon clear visualization, volvulus of the cecum.

The surgical procedure consisted of fibrotic bands section and, in order to protect the extrahepatic bile tube, the section of the collapsed transversal colon $20 \mathrm{cms}$ prior to its habitual hepatic flexion. Also section of the terminal ileus, $30 \mathrm{cms}$ before ileocecal valve; and then dissection of dilated intestinal from hepatoduodenal ligament, stomach and duodenum.

After verifying hemostasis and integrity of hepatoduodenal ligament, a primary intestinal anastomosis ileus-transversal colon was performed (Figure 4).

After the surgical procedure, Parenteral Nutrition started with 1700 $\mathrm{kcal}$ in $2400 \mathrm{mls}$, by intravenous approach due to complex abdominal surgery and prolonged fasting. During the third day after surgery evolves with minor abdominal pain, still being unable to channel intestinal gas.

At the eight day of surgery presents good liquid intake tolerance, abdominal peristaltic noise and no abdominal distension so it progresses to the tenth day when a normal diet is started and afterwards having no abdominal pain and normal bowel movements, with less effort than before surgery.

At the eleventh day after surgery is discharged, and later, medical office follow-up showed no abdominal pain, distension or complications.

\section{Discussion}

Even that up to $80 \%$ of the cases of intestinal malrotation are presented within the first month of age, it can be present later in other pediatric patients or even in adults, usually with a medical history of repetitive abdominal pain, nausea or vomit, sometimes causing repetitive intestinal occlusion or subocclusion symptoms.

The case of the patient presented had a medical history of repetitive intestinal occlusion symptoms that were treated conservatively with good outcome, but being recidivist. It was until further imaging studies that the suspicion of cecal volvulus and intestinal malrotation was made, that surgery was indicated and performed. Coeval cecal volvulus and intestinal malrotation anomaly is a rare entity [8-10]. 


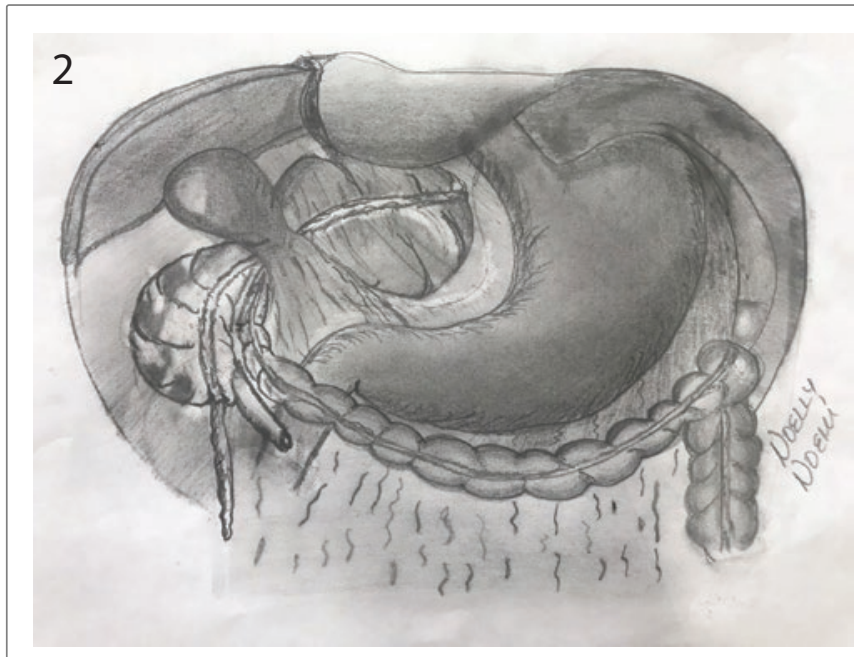

3

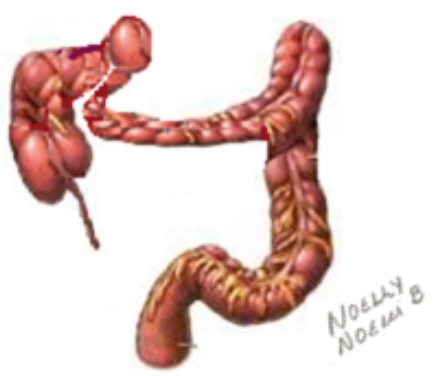

Figure 2 and 3: Short and ascendant cecum, behind hepatoduodenal ligament, dilated with transversal colon collapsed.

In adult patients, due to the absence of specific symptoms, surgical approach it is not usually offered or accepted, and less accepted by those patients with repetitive episodes managed successfully by conservative approach. The standard for diagnosis and reference regarding intestinal malrotation is the high-intestinal transit test, that can show an abnormal duodenum-jejunal union towards the right of the spinal vertebra $[7,11-13]$ however if it is no useful, an enema using barium can be of benefice [7,11-13].

After the volvulus diagnosis in an asymptomatic patient, some authors do not recommend a surgical approach due to the low intestinal ischemia incidence, but, not being able to predict those patients that will develop complications, all cases must be surgical approached [14-17].

Intestinal malrotation being a rare entity and an infrequent etiology of abdominal pain in adult patients, but it should be considered when associated medical history of repetitive intestinal occlusion symptoms. Regarding adult patients with repetitive events of intestinal occlusion, there are established paradigms of conservative management without considering medical history, and usually no further imaging studies.

In the reported case, surgery was offered due to the volvulus of the cecum found in CT scan, so we encourage to do a complete study and analysis of an adult patient with repetitive intestinal occlusion symptoms, in order to consider intestinal malrotation as a possible cause and be able to perform minimal invasive surgery as definitive treatment to avoid further complications, and have lower morbidity and mortality.
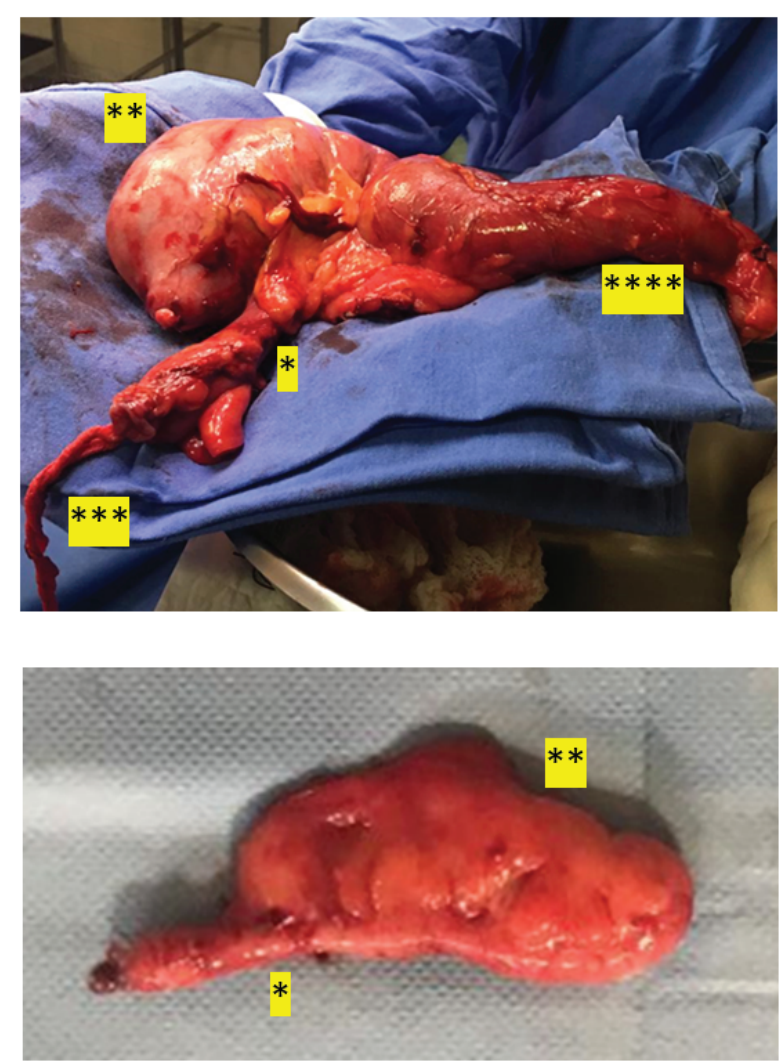

Figure 4: Surgical obtained piece consisting of the last $30 \mathrm{cms}$ of ileus*, cecum ${ }^{* *}$, appendix*** and proximal transverse colon****.

\section{Conclusion}

Intestinal malrotation, despite being infrequent in the adult patient, must be considered as a differential diagnosis in patients with a medical history of repetitive intestinal occlusion symptoms. Performing further study of this patients can facilitate the use of minimal invasive surgery as definitive treatment, and avoid complications such as a volvulus of the cecum, that force the need of major surgery with higher morbidity and mortality in adult patients.

\section{References}

1. Cisneros MO, Zárate PR, Gutiérrez SV, Rivera A, De los Ríos-Senmache $R$, et al. (2006) Malrotación intestinal en el adulto: Reporte de 1 caso y Revisión de la literura. Revista de Gastroenterología del Perú 26: 395-399.

2. Ladd WE (1936) Surgical diseases of the alimentary tract in infants. N Engl J Med 215: 705-708.

3. Kreindel T (2016) Anomalías de la rotación. Hospital Italiano de Buenos Aires.

4. Reyes BES, Castelló GM, Armas PBA (2012) Oclusión duodenal por malrotación intestinal en el adulto. AMC 16: 318-327.

5. Kantor JL (1934) Anomalies of the colon: Their Roentgen Diagnosis and Clinical Significance. Radiology 23: 651-662.

6. Pickhardt PJ, Bhalla S (2002) Intestinal malrotation in adolescents and adults: spectrum of clinical and imaging features. AJR Am J Roentgenol 179: 1429-1435. 
7. Tobias JD, Holcomb GW $3^{\text {rd }}$, Brock JW $3^{\text {rd }}$, Deshpande JK, Lowe S, et al. (1995) Cardiorespiratory changes in children during laparoscopy. J Pediatr Surg 30: 33-36.

8. Strouse PJ (2004) Disorders of intestinal rotation and fixation (“malrotation"). Pediatr Radiol 34: 837-851.

9. Torres AM, Ziegler MM (1993) Malrotation of the intestine. World J Surg 17: 326-331.

10. Jayathillake A, Shields MA (2005) Malrotation with volvulus: a rare cause of acute bowel obstruction in an adult. ANZ J Surg 75: 831-833.

11. Kapfer SA, Rappold JF (2004) Intestinal malrotation-not just the pediatric surgeon's problem. J Am Coll Surg 199: 628-635.

12. Long FR, Kramer SS, Markowitz RI, Taylor GE (1996) Radiographic patterns of intestinal malrotation in children. Radiographics 16: 547556.
13. Gupta AK, Guglani B (2005) Imaging of congenital anomalies of the gastrointestinal tract. Indian J Pediatr 72: 403-414.

14. Berrocal T, Lamas M, Gutierrez J, Torres I, Prieto C, et al. (1999) Congenital anomalies of the small intestine, colon and rectum. Radiographics 19: 1219-1236.

15. Keith JC, Thomas JJ, Buday SJ, Price PD, Smear J (2003) Asymptomatic midgut rotational anomalies in adults: 2 case reports and review of the literature. Contemp Surg 59: 322-325.

16. Janzen R, Sullivan B, Schmidt N (1999) Soft-tissue images. Intestinal nonrotation in the adult. Can J Surg 42: 248-249.

17. Sing RF, Blasko EC, Kefalides PT, Wolferth CC (1994) Management of anomalous rotation in adults. Am Surg 60: 938-941. 